\title{
Tuning the magnetic anisotropy in single-layer crystal structures
}

\author{
E. Torun, ${ }^{1,{ }^{*}}$ H. Sahin,,${ }^{1}$ C. Bacaksiz,${ }^{2}$ R. T. Senger, ${ }^{2}$ and F. M. Peeters ${ }^{1}$ \\ ${ }^{1}$ Department of Physics, University of Antwerp, Groenenborgerlaan 171, 2020 Antwerp, Belgium \\ ${ }^{2}$ Department of Physics, Izmir Institute of Technology, 35430 Izmir, Turkey
}

(Received 2 July 2015; revised manuscript received 25 August 2015; published 10 September 2015)

\begin{abstract}
The effect of an applied electric field and the effect of charging are investigated on the magnetic anisotropy (MA) of various stable two-dimensional (2D) crystals such as graphene, $\mathrm{FeCl}_{2}$, graphone, fluorographene, and $\mathrm{MoTe}_{2}$ using first-principles calculations. We found that the magnetocrystalline anisotropy energy of Co-on-graphene and Os-doped-MoTe 2 systems change linearly with electric field, opening the possibility of electric field tuning MA of these compounds. In addition, charging can rotate the easy-axis direction of Co-on-graphene and Os-doped-MoTe $\mathrm{M}_{2}$ systems from the out-of-plane (in-plane) to in-plane (out-of-plane) direction. The tunable MA of the studied materials is crucial for nanoscale electronic technologies such as data storage and spintronics devices. Our results show that controlling the MA of the mentioned 2D crystal structures can be realized in various ways, and this can lead to the emergence of a wide range of potential applications where the tuning and switching of magnetic functionalities are important.
\end{abstract}

DOI: 10.1103/PhysRevB.92.104407

PACS number(s): 73.22.-f, 68.43.Fg, 75.30.Gw

\section{INTRODUCTION}

One of the potential usages of monolayer materials such as graphene [1,2] and semiconductor transition metal dichalcogenides (sTMDs) [3,4] is in the field of spintronics [5-10]. One of the main limiting factors for a spin-electronic device are spin-flip transitions due to thermal fluctuations at room temperature. In order to preserve the magnetic moments from these fluctuations, a large magnetic anisotropy (MA) is essential [11-16]. Therefore, looking for materials with large MA is of importance for spintronics applications [7] and data storage [17]. Recent studies have shown that the magnetic moment and the MA in monolayer and bulk materials can be induced, modified, and tuned using an electric field ( $E$ field) [18-21], surface charging [22-25], applying biaxial strain [26-29], doping transition metal and other atoms [30,31], and dimers [32,33].

The microscopic origin of the MA is spin-orbit coupling (SOC), and therefore the possibility of manipulating the orbital and spin moment of a crystal is essential. In early studies, the MA of bulk and sandwich structures composed of thin layers were mainly investigated. Carcia et al. [34] showed that periodic $\mathrm{Pd} / \mathrm{Co}$ layered structures have perpendicular MA when the thickness of the Co layer is less than $8 \AA$. In addition, Draaisma et al. [35] found that the preferred magnetization direction can be modified by changing the thickness of the layers in $\mathrm{Pd} / \mathrm{Co}$ and $\mathrm{Pd} / \mathrm{Fe}$ sandwich structures. Moreover, using tight-binding [36] and first-principles approaches, the anisotropy values of such multilayer structures have been reported which are in good agreement with experiments [37-39]. In addition, Klaer et al. [40] observed an anisotropy in the orbital moment of the martensitic $\mathrm{Ni}_{2} \mathrm{MnGa}$ system that is consistent with the easy-axis direction of the compound. Using first-principles methods, the MA of $5 d$ transition metal adsorbed graphene nanoflakes [41] and Li-based phosphates [42] were also investigated.

\footnotetext{
*engin.torun@uantwerpen.be
}

It has been shown that applying an $E$ field is an efficient way to tune the MA of a structure. Shiota et al. [43] observed voltage-induced switching of the magnetic easy axis between the in-plane and perpendicular directions in $\mathrm{Au}(001) / \mathrm{ultrathin}$ $\mathrm{Fe}_{80} \mathrm{Co}_{20}(001) / \mathrm{MgO}(001) /$ polyimide/indium tin oxide (ITO) junctions. Endo et al. [44] reported that the magnetization easy axis of the layered $\mathrm{Co}_{40} \mathrm{Fe}_{40} \mathrm{~B}_{20}$ sandwiched with $\mathrm{MgO}$ and Ta changes its direction to out of plane from in plane as the thickness was reduced, and, furthermore, the magnetization direction could be modified with an applied $E$ field. In addition, Weisheit et al. [21] demonstrated that the MA of iron-platinum $(\mathrm{FePt})$ and iron-palladium (FePd) compounds can be reversibly modified by an applied $E$ field. Firstprinciples calculations predicted that a large out-of-plane MA for the $\mathrm{Fe} / \mathrm{MgO}$ interface is possible while the MA of the material cannot be switched by an applied $E$ field [45]. Using first-principles calculations, it has been shown that the MA of Fe-Pt multilayers can also be tuned by surface charging [22].

In spite of these extensive investigations on the MA of bulk materials, the literature is quite sparse for two-dimensional (2D) materials. The pristine form of graphene and TMDs do not exhibit MA due to their nonmagnetic nature and crystal symmetry. However, recent studies have revealed that there are methods to induce MA also in these systems. It has been shown that the magnetic property of graphene with a topological line defect can be tuned with strain [46]. Santos et al. [26] showed that the spin moment of the Ni-doped graphene can be tuned with strain using first-principles calculations. They found that after a critical strain of $6.5 \%$, a sharp transition from a low spin state to a high spin state occurs. Strain-induced magnetism is also observed in $\mathrm{NbS}_{2}$ and $\mathrm{NbSe}_{2}$ [27]. It is also shown that the magnetic moment of $\mathrm{Co}$ atom on pyridinic $\mathrm{N}$-doped graphene can be controlled by biaxial strain [28]. Using first-principles calculations, it has been shown that the MA of the Fe/graphene system can be modified by charge injection [25].

In this paper, we investigate the effect of an applied $E$ field and charging on the magnetocrystalline anisotropy energy (MAE) of several stable and easy to fabricate 2D materials. The paper is organized as follows: Computational details are given in Sec. II. We show the effect of an external $E$ field on 
MAE in Sec. III. Charging-induced modifications in the MAE values are investigated in Sec. IV. Finally, we conclude our results in Sec. V.

\section{COMPUTATIONAL METHODOLOGY}

All the calculations were performed within the spinpolarized density functional theory (DFT) using projectoraugmented-wave (PAW) potentials and a plane-wave basis set as implemented in the Vienna $a b$ initio simulation package (VASP) $[47,48]$. The cutoff energy for the plane waves was chosen to be $500 \mathrm{eV}$. Perdew-Burke-Ernzerhof's version of the generalized gradient approximation (GGA) [49] was used to describe the exchange correlation density functional. Spin-polarized calculations were performed in all cases and atomic charges were calculated by usage of the Bader charge population analysis [50]. The Gaussian smearing method was employed for the total energy calculations and the width of the smearing was chosen as $0.05 \mathrm{eV}$. DFT plus the long-range dispersion correction (DFT+D2) [51] method was used to calculate the nonlocal correlation energies. Geometric structures were fully optimized to minimize each component of the interatomic Hellmann-Feynman forces until a precision of $10^{-4} \mathrm{eV} / \AA$ was reached. The pressure in the unit cell was kept below $1 \mathrm{kbar}$. The convergence criterion for energy was chosen to be $10^{-5} \mathrm{eV}$ between two consecutive steps. The conjugate gradient method was used to compute lattice constants and total energies. In order to prevent interlayer interactions within the periodic images, we used a vacuum spacing between adjacent layers of $16 \AA$.

In our approach, without inclusion of SOC into the calculations, there is no link between the crystalline structure and the direction of the magnetic moments of the material. Therefore, the total energy of the system is not affected by a general rotation of all spin moments. In this "collinear" case, the off-diagonal terms in the matrix form of the spin-polarized Kohn-Sham equation vanish.

In order to create this link, the spin-orbit Hamiltonian should be included into the calculation, which is

$$
H_{\mathrm{SO}}^{\alpha \beta}=\frac{\hbar^{2}}{\left(2 m_{e} c\right)^{2}} \sum_{i, j}\left\langle\phi_{i}\left|\frac{1}{r} \frac{d V_{\text {spher }}}{d r}\right| \phi_{j}\right\rangle\left|\tilde{p}_{i}\right\rangle \vec{\sigma}^{\alpha \beta} \cdot \vec{L}_{i j}\left\langle\tilde{p}_{j}\right| .
$$

Inclusion of this term into the calculation mixes the spin-up and spin-down spinors and introduces off-diagonal terms to the matrix form of the spin-polarized Kohn-Sham equation.

The total energy of some condensed matter systems may change depending on the external magnetic field direction, which is defined as the MA of the material. The MAE of the systems can be calculated using the following expression,

$$
\mathrm{MAE}=E_{\|}-E_{\perp},
$$

where $E_{\|}$and $E_{\perp}$ are the total energies for the in-plane and outof-plane magnetization directions with respect to the surface of the single-layer structures, respectively. In order to find these two energy values, a relaxation procedure was performed in order to make the forces on the atoms and the pressure on the unit cell smaller than the threshold values. Then, the charge density which would be used in the noncollinear calculations was obtained by performing a collinear spin- polarized calculation. In the noncollinear calculations, the pre-converged charge and magnetization densities are used and non-self-consistent calculations were performed for the in-plane and out-of-plane magnetization directions.

The negative (positive) MAE corresponds to an easy axis along the in-plane (out-of-plane) direction with respect to the surface of the single-layer structures. For a few systems, we provide the difference in orbital moments $\left(\Delta L=L_{z}-L_{x}\right)$ of the doped or adsorbed atoms for different magnetization directions. In our calculations there is only one nonvanishing component of orbital moment which is in the direction of the spin quantization axis. So, the $L_{z}\left(L_{x}\right)$ value means the $z(x)$ component of the orbital moment when the quantization axis is in the out-of-plane (in-plane) direction.

The specific direction of the easy axis is determined by the geometric structure, bonding properties, characteristics of the valence and conduction band-edge states, as well as the strength of the SOC $[36,52,53]$. To establish a proper explanation for the easy-axis direction, we investigate these features of the compounds throughout this paper.

Our investigation on manipulating MAE covers five qualitatively different but complementary materials, considering their being symmetric or asymmetric, in- or out-of-plane easy-axis direction, magnetic or nonmagnetic, and added or substituted atoms. The optimized crystal structure and the ground state properties of these materials are given in Fig. 1 and Table I, respectively.

The first system that we study is Co on graphene, as shown in Fig. 1(a). This adsorption breaks the symmetry in out-of-plane direction. Our calculations show that the most favorable position for the Co atom is the center of the hexagon of graphene with a binding energy of $1.6 \mathrm{eV}$. The system has a $1.00 \mu_{B}$ magnetic moment and $3.68 \mathrm{meV}$ MAE per unit cell. It was experimentally found that a $\mathrm{Co}$ atom adsorbed on a graphene layer which is placed on top of a $\operatorname{Pt}(111)$ substrate has a magnetic anisotropy of $8.10 \pm 0.40 \mathrm{meV}$ [54]. Our calculations agree with this observation in the direction of the easy axis, yet the measured value is larger than the calculated MAE of $3.68 \mathrm{meV}$. This is one of the reasons behind the selection of this system in our study, to compare our results with the experimental data and test our formalism.

As the second system we investigate the MA properties of monolayer $\mathrm{FeCl}_{2}$ [see Fig. 1(b)]. It has been shown recently that the $\mathrm{FeCl}_{2}$ has a stable monolayer structure in the $1 T$ phase with a half-metallic ferromagnetic ground state [55]. Since it has a relatively large magnetic moment in its ground state, the $\mathrm{FeCl}_{2}$ monolayer is an interesting compound to investigate. The $2 \mathrm{D} \mathrm{FeCl}_{2}$ crystal in the $1 T$ phase is a magnetic system with $4.00 \mu_{B}$ per unit cell, according to our calculations. The calculated MAE of the system is $0.07 \mathrm{meV}$, which is a relatively small value compared to the Co-on-graphene case.

Another system that we study in this paper is graphone [see Fig. 1(c)], which is formed by $\mathrm{H}$ atoms covalently bonded on one of the sublattices of graphene and that was reported to be stable experimentally and theoretically $[56,57]$. The nonterminated $\mathrm{C}$ atom is the source of the magnetization of graphone. The net magnetic moment of graphone is $1.00 \mu_{B}$ per unit cell. However, our calculations show that graphone does not have a MA in its ground state in contrast to $\mathrm{FeCl}_{2}$. The difference in MAE of these similar compounds is due to the shapes 

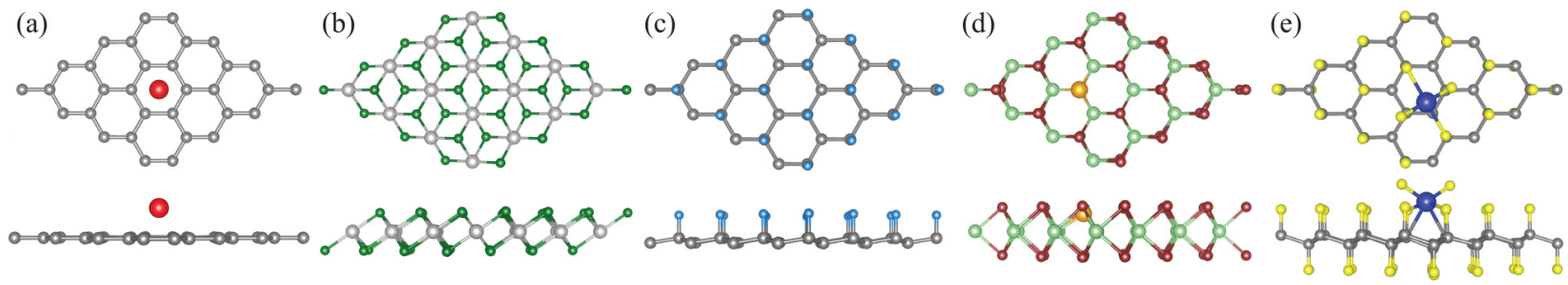

FIG. 1. (Color online) (a) Co on graphene. Co and $\mathrm{C}$ atoms are shown in red and dark gray, respectively. (b) $\mathrm{FeCl}_{2}$ in a $1 T$ structure. $\mathrm{Fe}$ and $\mathrm{Cl}$ atoms are shown in light gray and green, respectively. (c) Graphone. $\mathrm{C}$ and $\mathrm{H}$ atoms are shown in dark gray and blue, respectively. (d) Os-doped $\mathrm{MoTe}_{2}$. Mo, Te, and Os atoms are shown in light green, red, and orange, respectively. (e) V on fluorographene. F, C, and V atoms are shown in yellow, dark gray, and blue, respectively.

of the orbitals which are responsible for magnetization. In graphone, the origin of the magnetization is the $s$ orbital which is spherical symmetric and does not lead to anisotropy. On the other hand, in $\mathrm{FeCl}_{2}$, the $d$ orbitals are responsible for the magnetization, which are not spherically symmetric and which are responsible for the small anisotropy in this compound.

The next system that we study is Os-doped $\mathrm{MoTe}_{2}$. The reason behind the selection of this compound is that this is a representative of semiconductor $M X_{2}$ type compounds and it is the most recently synthesized one [58]. In the optimized geometry of the structure, the Os atom is localized at the site of a missing Te atom but positioned closer to the sublayer of Mo atoms shown in Fig. 1(d). The binding energy of the Os atom in this configuration is around $1.5 \mathrm{eV}$. The system has a $2.00 \mu_{B}$ magnetic moment per unit cell and the MAE is $-14.92 \mathrm{meV}$.

The last system we will investigate is a $\mathrm{V}$ atom on fluorographene (FG). Fluorographene is considered to be the 2D counterpart of Teflon and is a very stable compound [59]. In $\mathrm{V}$-adsorbed fluorographene, the $\mathrm{V}$ atom pulls two $\mathrm{F}$ atoms out of the surface, moves under them, and also tilts two second nearest neighboring $\mathrm{F}$ atoms towards itself [see Fig. 1(e)]. The binding energy of the $\mathrm{V}$ atom is around $7 \mathrm{eV}$ in this configuration. The system has a $1.00 \mu_{B}$ magnetic moment in the unit cell and the MAE is $-0.14 \mathrm{meV}$.

The systems in this paper have been selected on purpose, so before going into the result sections, we would like to reiterate briefly the purposes behind the selection of these systems. Co on graphene was previously studied experimentally, and therefore it provides us with a good test case to check whether or not our methodology and DFT are capable of correctly predicting the magnetic $\mathrm{MA}$. $\mathrm{FeCl}_{2}$ is a ferromagnetic monolayer structure with a relatively large magnetic moment and the

TABLE I. Lattice parameter $(a)$, charge donated $(\rho)$ by added or substituted atom, net magnetic moment $\left[\mu\left(\mu_{B}\right)\right]$, and magnetic anisotropy energy (MAE) for various 2D systems.

\begin{tabular}{lcccr}
\hline \hline & $\begin{array}{c}a \\
(\AA)\end{array}$ & $\begin{array}{c}\rho \\
(e)\end{array}$ & $\begin{array}{c}\mu \\
\left(\mu_{B}\right)\end{array}$ & $\begin{array}{r}\text { MAE } \\
(\mathrm{meV})\end{array}$ \\
\hline $\mathrm{Co}$ on $4 \times 4 \mathrm{G}$ & 9.90 & 0.60 & 1.00 & 3.68 \\
$\mathrm{FeCl}_{2}$ & 3.53 & $\mathrm{NA}$ & 4.00 & 0.07 \\
Graphone $_{\text {Os-doped MoTe }}$ & 2.53 & NA & 1.00 & 0.00 \\
$\mathrm{~V}$ on 4 4 FG & 14.15 & -0.40 & 2.00 & -14.92 \\
\hline \hline
\end{tabular}

origins of magnetism are the $d$ orbitals. Because ferromagnetic monolayer compounds are not very common, it is interesting to study the MA behavior of such materials. Graphone is also a ferromagnetic monolayer, but it has a smaller magnetic moment than $\mathrm{FeCl}_{2}$ and, furthermore, the $s$ orbitals are the origins of magnetism. This provides us with a good comparison with the very different case of $\mathrm{FeCl}_{2}$. Os-doped $\mathrm{MoTe}_{2}$ is a representative of a semiconductor $M X_{2}$ type of structure and is the most recently synthesized one. FG is considered to be the 2D counterpart of Teflon and it is a very stable compound. In addition, the binding energy of the $\mathrm{V}$ atom on FG is also very large and the optimized geometry is unique, i.e., the symmetry of the structure is broken.

\section{EFFECT OF ELECTRIC FIELD}

In this section the effect of a perpendicular $E$ field on the MAE is discussed. The $E$-field values were chosen to be compatible with earlier experimental and theoretical works [21,60-62]. The MAE and the magnetic moment of the structures with respect to the $E$ field are presented in Fig. 2. The positive (negative) values of the $E$ field in the figure correspond to the $+z(-z)$ direction.

Notice that there is almost a linear dependence between the external $E$ field and the MAE of the Co-on-graphene system which is shown in Fig. 2. It is obvious that in this system that the Co atom is the source of the magnetization and the MA. In order to reveal the origin of this linear relationship, the presence of occupied $d$ orbitals as well as the anisotropy in the orbital moment for different magnetization directions are useful.

In Fig. 3(a) we show the partial density of states (PDOS) of the $d$ orbitals of the Co atom on graphene. The shades of color represent the strength of the $E$ field (light, mid, and dark: $-0.50,0$, and $0.50 \mathrm{~V} / \AA$, respectively). In the neutral form of the system the $d_{x z}$ and $d_{y z}$ orbitals are partially occupied and the other $3 d$ orbitals of the Co atom are completely occupied. The PDOS characteristic of the Co atom does not change much with $E$ field. Therefore, to explain the linear trend in MAE, it is necessary to investigate also the other properties of the system.

In Fig. 2(c) the change in $\Delta L$ is shown. The $z$ component of the orbital moment is larger than the $x$ component of the orbital moment. So, $\Delta L$ has a positive value which means that the energy contribution of the Co atom to the spin-orbit energy is larger when the magnetization axis is in the out-of-plane direction, as can be seen in Fig. 2(c). This makes the total energy of the system lower when the magnetization axis is 

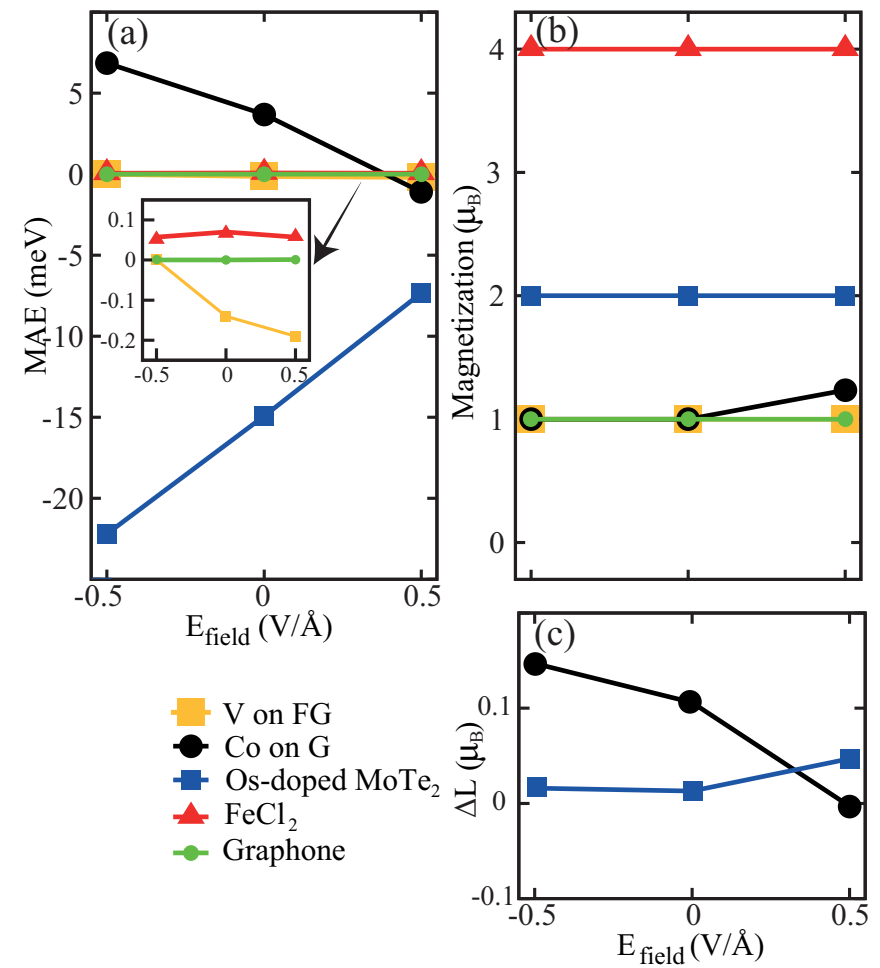

FIG. 2. (Color online) $E$-field dependence of (a) MAE, (b) magnetic moment for the considered systems, and (c) $\Delta L$ values for Co on graphene and Os-doped $\mathrm{MoTe}_{2}$. The inset in (a) is a zoomed view of $\mathrm{V}$ on $\mathrm{FG}, \mathrm{FeCl}_{2}$, and graphone.

in the out-of-plane direction, which is consistent with having a positive MAE for the neutral case of Co on graphene. In the case of the $-0.50 \mathrm{~V} / \AA E$ field, this difference is even larger, so this leads to a larger MAE than in the neutral case. On the other hand, when the $E$ field is $0.50 \mathrm{~V} / \AA$, the $\Delta L$ becomes negative and consistently the MAE of the system is negative. This shows that there is a direct relation between the easy-axis direction and the size of the orbital moment of the Co-on-graphene system. This prediction is consistent with the earlier paper by Bruna et al. in which the correlation between orbital moment and the MAE was shown for some

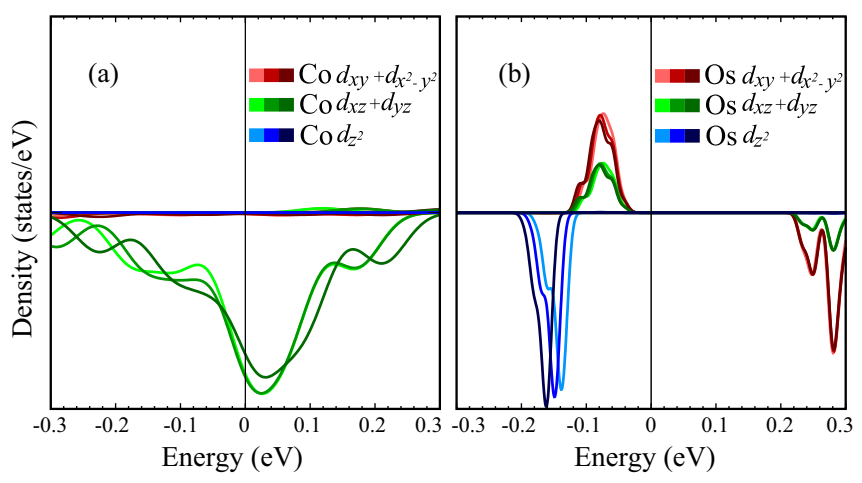

FIG. 3. (Color online) PDOS of the $d$ orbitals of (a) Co atom on a $4 \times 4$ graphene $(\mathrm{G})$ layer, and (b) Os-doped $\mathrm{MoTe}_{2}$ for different $E_{\text {field }}$ values: $-0.50,0$, and $0.50 \mathrm{~V} / \AA$ are represented by light, mid, and dark shades, respectively. The first and second channels for each system represent spin-up and spin-down DOS, respectively.
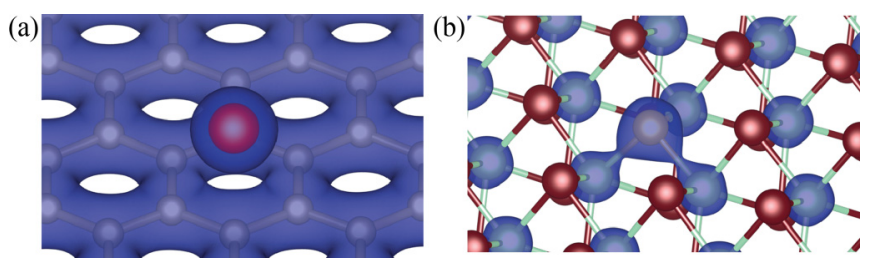

FIG. 4. (Color online) Total charge density of (a) Co on graphene and (b) Os-doped $\mathrm{MoTe}_{2}$. The isovalue is set to $0.09 e / \AA^{3}$ in both figures.

transition metal monolayers [36]. The almost linear relation that we obtained for $\Delta L$ as a function of $E_{\text {field }}$ can also be seen in Fig. 2(c) for the Co-on-graphene system.

The Os-doped $\mathrm{MoTe}_{2}$ is the other system for which the MA can be tuned with the $E$ field. The MAE of the compound changes linearly with the $E$ field which is shown in Fig. 2(a), which is similar to Co on graphene. The PDOS of the Os atom can give an idea about the nature of this linear dependence. In Fig. 3(b) the behavior of the $d$ orbitals for different $E$-field values is more clear than that for the Co-on-graphene system. The $d_{x^{2}}, d_{x y}, d_{x z}$, and $d_{x z}$ orbitals are almost unchanged for different $E$-field values, while the $d_{z^{2}}$ orbital moves away from the Fermi level when the $E$ field is $0.50 \mathrm{~V} / \AA$, and is close to the Fermi level when the $E$ field is $-0.50 \mathrm{~V} / \AA$. This linear trend also shows that it is possible to switch the preferable alignment of the magnetization for higher $E$-field values if the structure is not modified. As can be seen from the figure, the $d_{z^{2}}$ orbital is more sensitive to the $E$ field than the other $d$ orbitals. The reason is that the $d_{z^{2}}$ orbitals have an out-of-plane character and the direction of the external $E$ field in our calculations is perpendicular to the surface of the monolayers.

The $\Delta L$ value as a function of the $E$ field is given in Fig. 2(c). Os has covalent bonding with the $\mathrm{MoTe}_{2}$ monolayer, which may destroy the information about the orbital moments. On the other hand, there is no covalent bonding between $\mathrm{Co}$ and the graphene layer, as can be seen in Fig. 4. This means that Co on graphene and Os-doped $\mathrm{MoTe}_{2}$ are completely different systems in terms of bonding characteristics, and, therefore, the origins of the MA in these materials are completely different.

Our calculations reveal that if the material does not possess MA in its ground state, such as graphone, an $E$ field cannot induce anisotropy to the system. If the material has a relatively small MAE such as $\mathrm{FeCl}_{2}$ and V-doped fluorographene, the effect of the $E$ field on MA is relatively small as compared to the Co-on-graphene and Os-doped-MoTe ${ }_{2}$ cases. For example, the $\mathrm{FeCl}_{2}$ monolayer has $0.07 \mathrm{meV} \mathrm{MA}$ in its ground state and applying -0.5 and $0.5 \mathrm{~V} / \AA$ external $E$ fields changes it to $0.057 \mathrm{meV}$ for both cases. For V-doped fluorographene, the system has $-0.14 \mathrm{meV}$ MA in its ground state and applying -0.5 and $0.5 \mathrm{~V} / \AA$ external $E$ fields changes it to 0 and $-0.19 \mathrm{meV}$, respectively.

\section{EFFECT OF CHARGING}

Charging is one of the most practical methods to manipulate the charge distribution and consequently the magnetic property of a material. Therefore, it is expected to modify the degree of MA as well when we vary the Fermi energy. In this section we 

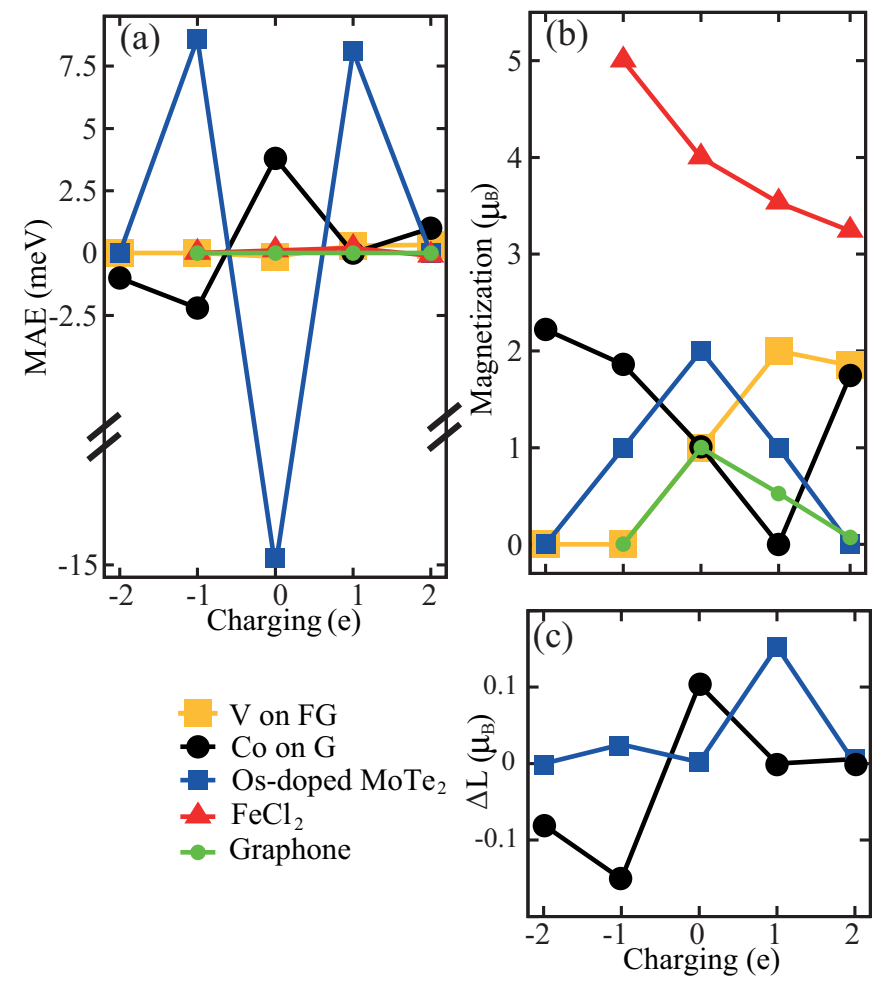

FIG. 5. (Color online) Charging dependence of (a) MAE, (b) magnetic moment for the considered systems, and (c) $\Delta L$ values for Co on graphene and Os-doped $\mathrm{MoTe}_{2}$.

investigate the consequences of charging on the MAE of the different 2D monolayer systems shown in Fig. 1. The MAE and the net magnetic moments of the structures with respect to charging are shown in Fig. 5.

Charging of $\mathrm{Co}$ on graphene changes remarkably its magnetic state and the MA. Adding one electron makes the system nonmagnetic and consequently leads to vanishing MAE. A Bader analysis shows that the charge of the Co atom becomes $8.60 e$, which was $8.40 e$ in the neutral form. In this configuration all the $3 d$ orbitals of the Co atom become occupied, as can be seen in Fig. 6. Adding two electrons makes the magnetic moment of the system $2.00 \mu_{B}$ and creates a positive MAE, of almost $1 \mathrm{meV}$. The Bader analysis shows that the charge of the Co atom is $9.20 e$, which indicates that most of the extra charge is located on the Co atom. The PDOS of this configuration and of the neutral system show similarities: The out-of-plane oriented $d_{x z}$ and $d_{y z}$ orbitals are partially occupied and the other $3 d$ orbitals of the Co atom are completely occupied. This similarity results in the same easy-axis direction with the neutral system, which is the out-of-plane direction.

Removing one electron makes the magnetic moment of the system around $2.00 \mu_{B}$ and changes the sign of the MAE. A Bader analysis shows that the charge of the Co atom is $8.20 e$, which indicates that a small part of the removed charge is from the Co atom. Our PDOS analysis shows that the partially occupied out-of-plane $d_{x z}$ and $d_{y z}$ orbitals become more unoccupied and the in-plane orbitals become closer to the Fermi level (see Fig. 6), which could be the reason for the change in sign of the MAE of the system. Removing

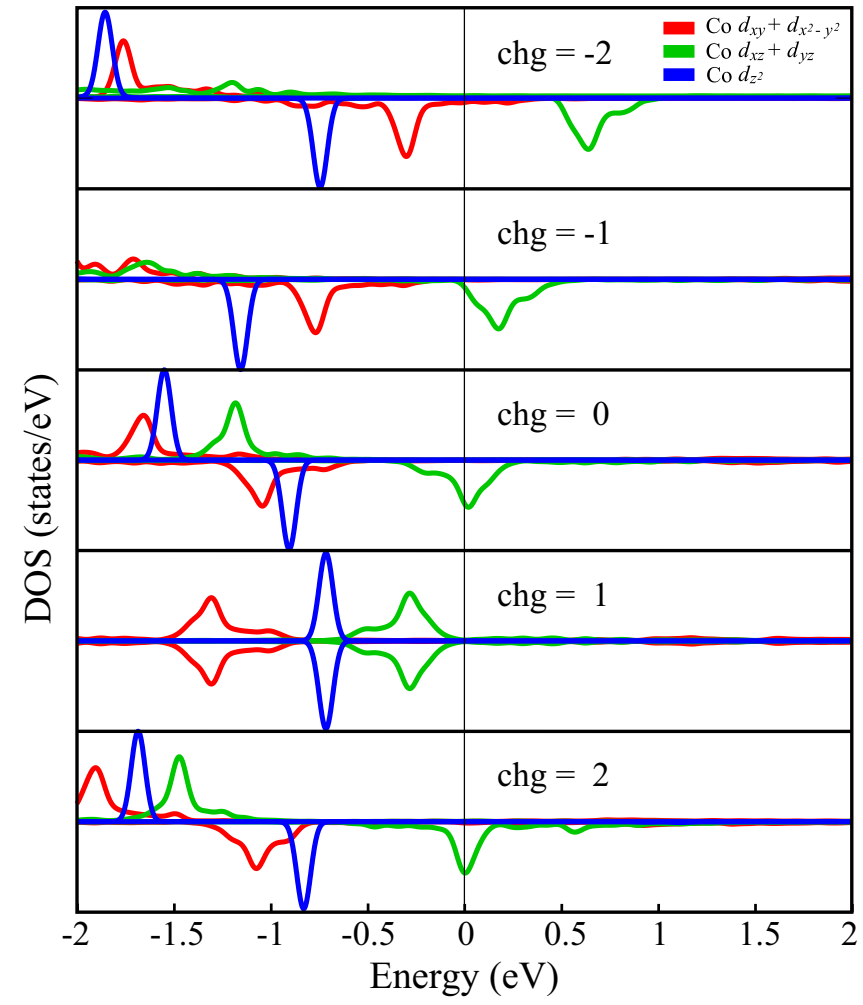

FIG. 6. (Color online) PDOS of the $d$ orbitals of Co atom on a $4 \times 4$ graphene layer for different charging cases. The first and second channels for each charging case represent spin-up and spin-down DOS, respectively.

two electrons makes the magnetic moment and the MAE of the system around $2.20 \mu_{B}$ and $-1 \mathrm{meV}$, respectively. In this configuration, the charge of the Co atom is $8.40 e$, which is almost the same value compared with the neutral form of the system.

As mentioned earlier, the MA of a compound depends strongly on the size of the orbital moment. We found a correlation with the direction of the easy axis and the size of the orbital moment for the Co-on-graphene system for different charging cases [see Fig. 5(c)]. For the neutral case, our calculations show that the orbital moment along the $z$ direction is larger than the value for the $x$ direction, $0.22 \mu_{B}$ and $0.12 \mu_{B}$, respectively. Consistently, the easy-axis direction of this case is along the $z$ direction, which is along the direction of the larger orbital moment. For the $2 e$ added case, the orbital moment along the $z$ direction is still larger than the value for the $x$ axis, $0.14 \mu_{B}$ and $0.13 \mu_{B}$, respectively. The direction of the easy axis is still in the $z$ direction with a smaller MAE compared with the neutral case. This is due to the smaller difference between the orbital moment along the different directions. As seen in Fig. 5, the direction of the easy axis changes when electrons are removed from the system. Our calculations show that the orbital moment of these cases along the $x$ direction is larger than the value along the $z$ direction. This is consistent with our earlier interpretations.

Another interesting prediction is that there is an alteration in the distance of the Co atom to the graphene layer for different charging cases. For the neutral system the vertical distance of the Co atom to the graphene layer is around $1.50 \AA$. Adding 
one electron makes the Co atom closer to the graphene layer; the distance becomes $1.45 \AA$ in this case. Adding two electrons makes the atom move away from the layer; the distance becomes $1.60 \AA$. Removing one or two electrons has the same effect; the Co atom graphene layer distances are 1.60 and $1.57 \AA$ A, respectively.

As discussed earlier, the $\mathrm{FeCl}_{2}$ monolayer carries a magnetic moment of $4.00 \mu_{B}$ per unit cell and has almost $0.07 \mathrm{meV}$ MAE in its neutral form. This gives further insight into understanding the essential requirements to have MA in a material. Having a large net magnetic moment alone does not give rise to a large MA. As seen in Fig. 5, the magnetic moment of the system changes with charging, however, charging cannot induce MA to the $\mathrm{FeCl}_{2}$ monolayer. When two electrons are removed from this system, the calculation did not converge to a reasonable geometry, and therefore we left out this case from our discussion.

Similar to the $\mathrm{FeCl}_{2}$ monolayer, graphone carries a net magnetic moment in the unit cell but does not have MA in its neutral form. This is another example that shows that having only a net magnetic moment does not give rise to MA. Charging modifies the magnetic moment of graphone but cannot induce MA, as seen in Fig. 5. For the case of two-electron removal, the calculation did not converge to a reasonable geometry such as in the case for $\mathrm{FeCl}_{2}$, and therefore we left out this case from the discussions.

Os-doped $\mathrm{MoTe}_{2}$ is the only system which has an in-plane easy axis in its neutral form. Adding one electron makes the magnetic moment of the system $1.00 \mu_{B}$ and changes the sign

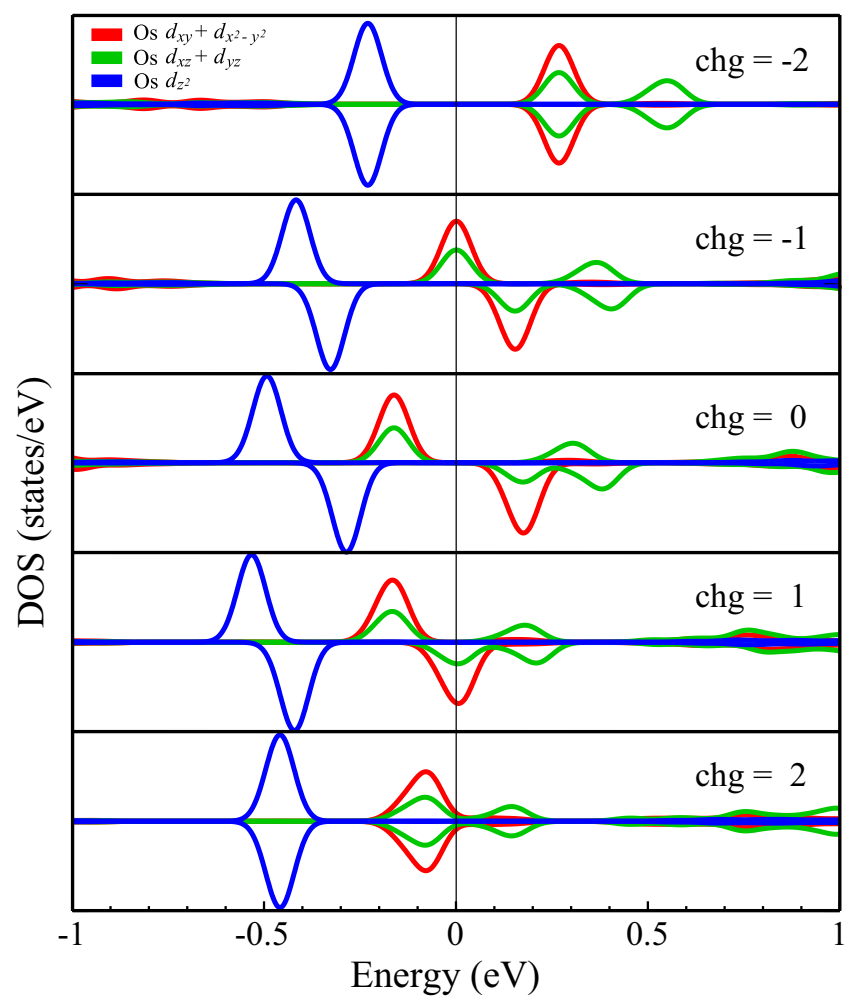

FIG. 7. (Color online) PDOS of the $d$ orbitals of Os-doped $\mathrm{MoTe}_{2}$ for different charging cases. The first and second channels for each charging case represent spin-up and spin-down DOS, respectively. of the MAE of the system. In this case, the PDOS of the system (see Fig. 7) shows that $d_{x z}$ and $d_{y z}$ orbitals as well as $d_{x^{2}}$ and $d_{x y}$ start to become occupied. A Bader analysis shows that a small amount of extra charge is localized on the Os atom. Removing one electron has similar consequences; the magnetic moment of the system becomes $1.00 \mu_{B}$ and the sign of the MAE changes as compared to its neutral form. The PDOS of this configuration shows that almost all the orbitals except $d_{z^{2}}$ become unoccupied. The PDOS of the orbitals in the vicinity of the Fermi level has similar characteristics with one electron added, so these two cases have the same easyaxis direction. This shows that the easy-axis direction clearly depends on the characteristics of the orbitals in the vicinity of the Fermi level. Similar to the discussions that we had in the $E$ field section, there is not a one-to-one correlation between the orbital moment and the MA of the Os-doped-MoTe 2 system.

We found that charging changes the magnetic state of the V-doped flourographene, however, the small MA and the optimized geometry of the system are almost independent of it. Our investigation of the MA of various systems showed that charging can switch the easy-axis direction of the Co on graphene and Os-doped $\mathrm{MoTe}_{2}$. However, the zero or small MAEs of the other systems are not affected by charging, even though their magnetic state is altered by it.

\section{CONCLUSION}

In summary, we have investigated the modification in the MA of several typical and easy to fabricate 2D monolayer crystal structures with an applied external $E$ field and charging. It is seen that the MA of a material depends strongly on the broken crystal symmetry and the orbital character of the states in the vicinity of the Fermi level as well as the size of the $\mathrm{SOC}$ in the crystal. We have shown that the presence of a large net magnetic moment itself is not enough to induce MA in a material, which is the reason why $\mathrm{FeCl}_{2}$ has a small MA and graphone does not possess MA. The SOC strength of the Co and $\mathrm{Fe}$ atoms are comparable to each other, but the MAE of Co on graphene and $\mathrm{FeCl}_{2}$ is relatively large. The reason could be the broken crystal symmetry of the Co-on-graphene system, which enhances the MA of a material.

Our calculations revealed that the MA of Co on graphene and Os-doped $\mathrm{MoTe}_{2}$ can be tuned by an $E$ field and by charging, and we have shown that the MAE of these systems changes linearly with applied $E$ field and can change its sign with charging. This prediction can be useful for the usage of these materials in spin electronics applications where tuning and switching functionalities are needed.

\section{ACKNOWLEDGMENTS}

This work was supported by the Flemish Science Foundation (FWO-Vl) and the Methusalem Foundation of the Flemish government. Computational resources were provided by TUBITAK ULAKBIM, High Performance and Grid Computing Center (TR-Grid e-Infrastructure), and HPC infrastructure of the University of Antwerp (CalcUA), a division of the Flemish Supercomputer Center (VSC), which is funded by the Hercules Foundation. H.S. is supported by a FWO Pegasus Marie Curie Fellowship. C.B. and R.T.S. acknowledge support from TUBITAK Project No. 111T318. 
[1] K. S. Novoselov, A. K. Geim, S. V. Morozov, D. Jiang, Y. Zhang, S. V. Dubonos, I. V. Grigorieva, and A. A. Firsov, Science 306, 666 (2004).

[2] A. K. Geim and K. S. Novoselov, Nat. Mater. 6, 183 (2007).

[3] J. N. Coleman, M. Lotya, A. O’Neill, S. D. Bergin, P. J. King, U. Khan, K. Young, A. Gaucher, S. De, R. J. Smith, I. V. Shvets, S. K. Arora, G. Stanton, H.-Y. Kim, K. Lee, G. T. Kim, G. S. Duesberg, T. Hallam, J. J. Boland, J. J. Wang, J. F. Donegan, J. C. Grunlan, G. Moriarty, A. Shmeliov, R. J. Nicholls, J. M. Perkins, E. M. Grieveson, K. Theuwissen, D. W. McComb, P. D. Nellist, and V. Nicolosi, Science 331, 568 (2011).

[4] C. Ataca, H. Sahin, and S. Ciraci, J. Phys. Chem. C 116, 8983 (2012).

[5] O. V. Yazyev and M. I. Katsnelson, Phys. Rev. Lett. 100, 047209 (2008).

[6] D. Xiao, G.-B. Liu, W. Feng, X. Xu, and W. Yao, Phys. Rev. Lett. 108, 196802 (2012).

[7] R. A. Lukaszew, Y. Sheng, C. Uher, and R. Clarke, Appl. Phys. Lett. 75, 1941 (1999).

[8] Y.-W. Son, M. L. Cohen, and S. G. Louie, Nature (London) 444, 347 (2006).

[9] S. A. Wolf, D. D. Awschalom, R. A. Buhrman, J. M. Daughton, S. von Molnár, M. L. Roukes, A. Y. Chtchelkanova, and D. M. Treger, Science 294, 1488 (2001).

[10] C. M. Fang, G. A. de Wijs, and R. A. de Groot, J. Appl. Phys. 91, 8340 (2002).

[11] D. S. Wang, R. Q. Wu, and A. J. Freeman, Phys. Rev. B 47, 14932 (1993).

[12] S. H. Charap, P. L. Lu, and Y. He, IEEE Trans. Magn. 33, 978 (1997).

[13] P. Gambardella, S. Rusponi, M. Veronese, S. S. Dhesi, C. Grazioli, A. Dallmeyer, I. Cabria, R. Zeller, P. H. Dederichs, K. Kern, C. Carbone, and H. Brune, Science 300, 1130 (2003)

[14] J. V. Barth, G. Costantini, and K. Kern, Nature (London) 437, 671 (2005).

[15] S. Ikeda, K. Miura, H. Yamamoto, K. Mizunuma, H. D. Gan, M. Endo, S. Kanai, J. Hayakawa, F. Matsukura, and H. Ohno, Nat. Mater. 9, 721 (2010).

[16] O. Mosendz, S. Pisana, J. W. Reiner, B. Stipe, and D. Weller, J. Appl. Phys. 111, $07 B 729$ (2012).

[17] J. M. Galloway, J. E. Talbot, K. Critchley, J. J. Miles, and J. P. Bramble, Adv. Funct. Mater. 25, 4590 (2015).

[18] Z. Bai, L. Shen, Y. Cai, Q. Wu, M. Zeng, G. Han, and Y. P. Feng, New J. Phys. 16, 103033 (2014).

[19] P. V. Ong, N. Kioussis, P. K. Amiri, J. G. Alzate, K. L. Wang, G. P. Carman, J. Hu, and R. Wu, Phys. Rev. B 89, 094422 (2014).

[20] A. S. Zyazin, J. W. G. van den Berg, E. A. Osorio, H. S. J. van der Zant, N. P. Konstantinidis, M. Leijnse, M. R. Wegewijs, F. May, W. Hofstetter, C. Danieli, and A. Cornia, Nano Lett. 10, 3307 (2010).

[21] M. Weisheit, S. Fähler, A. Marty, Y. Souche, C. Poinsignon, and D. Givord, Science 315, 349 (2007).

[22] P. Ruiz-Díaz, T. R. Dasa, and V. S. Stepanyuk, Phys. Rev. Lett. 110, 267203 (2013).

[23] P. Ruiz-Díaz and V. S. Stepanyuk, J. Phys. D: Appl. Phys. 47, 105006 (2014).

[24] O. O. Brovko, P. Ruiz-Díaz, T. R. Dasa, and V. S. Stepanyuk, J. Phys.: Condens. Matter 26, 093001 (2014).

[25] S. J. Gong, C.-G. Duan, Z.-Q. Zhu, and J.-H. Chu, Appl. Phys. Lett. 100, 122410 (2012).
[26] E. J. G. Santos, A. Ayuela, and D. Sánchez-Portal, J. Phys. Chem. C 116, 1174 (2011).

[27] Y. Zhou, Z. Wang, P. Yang, X. Zu, L. Yang, X. Sun, and F. Gao, ACS Nano 6, 9727 (2012).

[28] S. Jo, S. Lee, K.-H. Yun, Y. Hwang, and Y.-C. Chung, IEEE Trans. Magn. 50, 1 (2014).

[29] Q. Tang, Z. Zhou, and Z. Chen, Nanoscale 5, 4519 (2013).

[30] Q. Dubout, F. Donati, C. Wäckerlin, F. Calleja, M. Etzkorn, A. Lehnert, L. Claude, P. Gambardella, and H. Brune, Phys. Rev. Lett. 114, 106807 (2015).

[31] W. T. Cong, Z. Tang, X. G. Zhao, and J. H. Chu, Sci. Rep. 5, 9361 (2014).

[32] R. Xiao, D. Fritsch, M. D. Kuz'min, K. Koepernik, H. Eschrig, M. Richter, K. Vietze, and G. Seifert, Phys. Rev. Lett. 103, 187201 (2009).

[33] J. Hu and R. Wu, Nano Lett. 14, 1853 (2014).

[34] P. F. Carcia, A. D. Meinhaldt, and A. Suna, Appl. Phys. Lett. 47, 178 (1985).

[35] H. J. G. Draaisma, W. J. M. de Jonge, and F. J. A. den Broeder, J. Magn. Magn. Mater. 66, 351 (1987).

[36] P. Bruno, Phys. Rev. B 39, 865 (1989).

[37] G. H. O. Daalderop, P. J. Kelly, and M. F. H. Schuurmans, Phys. Rev. B 41, 11919 (1990).

[38] K. Kyuno, R. Yamamoto, and S. Asano, J. Phys. Soc. Jpn. 61, 2099 (1992).

[39] M. T. Johnson, P. J. H. Bloemen, F. J. A. den Broeder, and J. J. de Vries, Rep. Prog. Phys. 59, 1409 (1996).

[40] P. Klaer, T. Eichhorn, G. Jakob, and H. J. Elmers, Phys. Rev. B 83, 214419 (2011).

[41] I. Beljakov, V. Meded, F. Symalla, K. Fink, S. Shallcross, M. Ruben, and W. Wenzel, Nano Lett. 14, 3364 (2014).

[42] K. Yamauchi and S. Picozzi, Phys. Rev. B 81, 024110 (2010).

[43] Y. Shiota, T. Maruyama, T. Nozaki, T. Shinjo, M. Shiraishi, and Y. Suzuki, Appl. Phys. Express 2, 063001 (2009).

[44] M. Endo, S. Kanai, S. Ikeda, F. Matsukura, and H. Ohno, Appl. Phys. Lett. 96, 212503 (2010).

[45] K. Nakamura, T. Akiyama, T. Ito, M. Weinert, and A. J. Freeman, Phys. Rev. B 81, 220409(R) (2010).

[46] L. Kou, C. Tang, W. Guo, and C. Chen, ACS Nano 5, 1012 (2011).

[47] G. Kresse and D. Joubert, Phys. Rev. B 59, 1758 (1999).

[48] G. Kresse and J. Furthmüller, Phys. Rev. B 54, 11169 (1996).

[49] J. P. Perdew, K. Burke, and M. Ernzerhof, Phys. Rev. Lett. 78, 1396 (1997).

[50] R. F. W. Bader, Atoms in Molecules: A Quantum Theory (Oxford University Press, New York, 1994).

[51] S. Grimme, J. Comput. Chem. 27, 1787 (2006).

[52] J. Stöhr, J. Magn. Magn. Mater. 200, 470 (1999).

[53] G. van der Laan, J. Phys.: Condens. Matter 10, 3239 (1998).

[54] F. Donati, Q. Dubout, G. Autès, F. Patthey, F. Calleja, P. Gambardella, O. V. Yazyev, and H. Brune, Phys. Rev. Lett. 111, 236801 (2013).

[55] E. Torun, H. Sahin, S. K. Singh, and F. M. Peeters, Appl. Phys. Lett. 106, 192404 (2015).

[56] J. Zhou, Q. Wang, Q. Sun, X. S. Chen, Y. Kawazoe, and P. Jena, Nano Lett. 9, 3867 (2009).

[57] J. Zhou, M. M. Wu, X. Zhou, and Q. Sun, Appl. Phys. Lett. 95, 103108 (2009). 
[58] B. Chen, H. Sahin, A. Suslu, L. Ding, M. I. Bertoni, F. M. Peeters, and S. Tongay, ACS Nano 9, 5326 (2015).

[59] R. R. Nair, W. Ren, R. Jalil, I. Riaz, V. G. Kravets, L. Britnell, P. Blake, F. Schedin, A. S. Mayorov, S. Yuan, M. I. Katsnelson, H-M. Cheng, W. Strupinski, L. G. Bulusheva, A. V. Okotrub, I. V. Grigorieva, A. N. Grigorenko, K. S. Novoselov, and A. K. Geim, Small 6, 2877 (2010).
[60] H. Zhang, C. Lazo, S. Blügel, S. Heinze, and Y. Mokrousov, Phys. Rev. Lett. 108, 056802 (2012).

[61] S. Ishibashi, T. Tamura, S. Tanaka, M. Kohyama, and K. Terakura, Phys. Rev. B 76, 153310 (2007).

[62] Q. Liu, L. Li, Y. Li, Z. Gao, Z. Chen, and J. Lu, J. Phys. Chem. C 116, 21556 (2012). 